\title{
Desarrollo de habilidades comunicativas y estilos de aprendizaje VAK. Una experiencia con estudiantes de grado segundo de básica primaria*
}

Development of communicative skills and VAK learning styles. An experience with students of second grade of primary basics

Desenvolvimento de competências comunicativas e estilos de aprendizagem da VAK.

Uma experiência com os alunos do segundo grau de fundamentos primários*

William R. Avendaño C. **

Gerson Rueda V. ***

Torcoroma Rolón B. $* * * *$

Fecha de Recibido: Agosto 10 del 2018

Fecha de Aceptación: Diciembre 03 de 2018

Fecha de Publicación: Enero 01 de 2019

DOI: http://dx.doi.org/10.22335/rlct.v10i2.557

**Magister en Educación, Universidad de la Sabana. Filiación: Universidad Francisco de Paula Santander. Correo Electrónico: wrac2008@hotmail.com, williamavendaño@ufps.edu.co ORCID: 0000-0002-7510-8222

***Magister en Práctica Pedagógica, Universidad Francisco de Paula Santander. Filiación Universidad Francisco de Paula Santander. Correo electrónico: geruedavera@gmail.com

gersonruedavera@ufps.edu.co ORCID: 0000-0001-9032-7100 Frontera. Correo electrónico: torcorol@hotmail.com ORCID: 0000-0003-3685-3294

\section{Resumen}

Se muestran los resultados de una intervención realizada a estudiantes del grado segundo de básica primaria de una institución educativa rural del municipio de El Zulia (Colombia) para el desarrollo de habilidades comunicativas atendiendo a los estilos de aprendizaje visual, auditivo y kinestésico (VAK). La investigación se enmarca en el paradigma histórico hermenéuticointerpretativo, el enfoque cualitativo y un diseño investigación-acción. Participaron 13 estudiantes en edades comprendidas entre los 7 y 10 años. Se empleó como instrumento una prueba inicial y final 
de valoración del estilo de aprendizaje predominante. Los resultados muestran una mejora significativa en las habilidades comunicativas de los escolares al compararse la prueba inicial y final con posterioridad a una intervención efectuada sobre el grupo.

Palabras clave: Educación, aprendizaje, Aprendizaje verbal, Aprendizaje visual, Método de enseñanza, Enseñanza de la lengua materna.

\section{Abstract}

Show the results of an intervention made to students of the second grade of primary school of a rural educational institution of the municipality of El Zulia (Colombia) for the development of communication skills attending to the visual, auditory and kinesthetic (VAK) learning styles. The research is framed in the hermeneuticalinterpretative historical paradigm, the qualitative approach and a research-action design. 13 students participated in ages between 7 and 10 years old. An initial and final assessment test of the predominant learning style was used as an instrument. The results show a significant improvement in the communicative abilities of the students when comparing the initial and final test after an intervention carried out on the group.

Keywords: Education, Learning, Verbal learning, Visual learning, Teaching method, Mother tongue teaching.

\section{Resumo}

São apresentados os resultados de uma intervenção feita a alunos do segundo ano do ensino fundamental de uma instituição de ensino rural do município de El Zulia (Colômbia) para o desenvolvimento de habilidades de comunicação que atendem aos estilos de aprendizagem visual, auditivo e kinestesic (VAK). A pesquisa enquadrase no paradigma histórico hermenêuticointerpretativo, na abordagem qualitativa e no delineamento pesquisa-ação. 13 estudantes participaram de idades entre 7 e 10 anos. Um teste de avaliação inicial e final do estilo de aprendizagem predominante foi utilizado como instrumento. Os resultados mostram uma melhora significativa nas habilidades comunicativas dos estudantes quando comparados os testes inicial e final após uma intervenção realizada no grupo.

Palavras-chave: Educação, Aprendizagem, Aprendizagem verbal, Aprendizagem visual, Método de ensino, Ensino da língua materna.

\section{Introducción}

El lenguaje ostenta una gran importancia para la escuela pues tiene un doble valor: uno subjetivo, traducido en una herramienta cognitiva para el individuo y que le permite la aprehensión de la realidad, la diferenciación de los objetos y la diferenciación con los otros sujetos que lo rodean; y uno social que se concreta en el conocimiento de la realidad socio-cultural y la participación para la transformación de la misma por parte del individuo (Ministerio de Educación Nacional de Colombia, 2006, pág. 18). No hay duda sobre la trascendencia 
que tiene el lenguaje para el ser humano, y por ello Piaget (1991) expone que "el lenguaje es fuente del pensamiento" (pág. 111), el cual permite el desarrollo de un conjunto de operaciones lógicas y la posibilidad se socializar con los otros en medio de la cultura y los procesos de significación colectiva del mundo.

El lenguaje se convierte en la herramienta por excelencia mediante la cual el hombre construye y materializa su existencia, pues esta se encuentra ligada con la significación que tiene del mundo que habita. Por otro lado, el lenguaje ostenta una función social que posibilita la organización y cohesión de los hombres, la interacción, la cognición, el conocimiento y el mundo cultural como se conoce. El lenguaje no sólo se asume como medio de expresión, sino como elemento esencial del conocimiento, en cuanto es el lugar donde ocurre la significación. Piaget (1991) explica que el mismo lenguaje direcciona "conceptos y nociones que pertenecen a todos y que refuerzan el pensamiento individual mediante un amplio sistema de pensamiento colectivo" (pág. 34), y por tanto, es medio para el desarrollo del pensamiento y el acto de conocer.

Los primeros ciclos de formación escolar son determinantes en la vida del niño. Las habilidades comunicativas: hablar, escuchar, leer y escribir, se desarrollan y fortalecen en las primeras etapas escolares determinando el potencial de aprendizaje de los escolares. Cada una de estas habilidades tiene una trascendencia significativa en términos pedagógicos en la escuela, pues sí se entiende la escuela como un espacio de socialización, las habilidades comunicativas resultan indispensables y transversales en cada una de las prácticas pedagógicas, y del mismo modo, cada una depende de las otras en una mayor o menor medida: "si se contempla el aula como un espacio donde se desarrollan actividades discursivas diversas e interrelacionadas, se constata que las diferentes habilidades lingüísticas no se producen aisladamente y que su enseñanza implica la confluencia de todas ellas" (Camps, 2005, pág. 37).

$Y$ en efecto, todas las actividades que se realizan en la escuela exigen de una u otra manera las habilidades comunicativas. Al efectuar una exposición se debe leer, hablar, discutir, socializar, defender puntos de vista, aclarar dudas, entre otras; o cuando se resuelve un conjunto de ejercicios lógico-matemáticos se requiere escucha y atención, lectura juiciosa del problema, hablar en caso de dudas o inquietudes exigiendo la formulación precisa y concisa de preguntas; o en el momento de un conflicto y su solución se debe saber hablar y escuchar a los otros actores requiriendo tolerancia, paciencia y respeto. En todo el escenario escolar se encuentran las habilidades comunicativas y de allí que la comunicación sea una noción que se relaciona de manera directa con la educación y la pedagogía.

En el escenario escolar, las habilidades comunicativas son tanto un fin como un medio. Es un fin por cuanto la escuela debe promover habilidades y destrezas para la escucha, el habla, la lectura y la escritura, es decir, estas son objeto 
del aprendizaje, un objetivo de la enseñanza. Y es un medio toda vez que estas habilidades sirven como instrumento para el acceso a los otros campos del saber, convirtiéndose en herramientas para el mismo sujeto de formación. Por la importancia de las habilidades comunicativas, el docente de hoy se enfrenta a un reto significativo: lograr desde su acción pedagógica la adquisición y el desarrollo de las habilidades comunicativas en los estudiantes desde los primeros años de formación. Esta tarea implica, entre otras cosas, identificar la forma en que los niños llevan a cabo su proceso de aprendizaje dada sus particularidades y características, diseñar, implementar y evaluar las acciones pedagógicas adecuadas para este propósito, reconocer la naturaleza de cada una de las habilidades comunicativas de forma acertada, así como el carácter holístico, integral e interdependiente de las mismas.

Expuesto lo anterior, en este estudio se socializan los resultados de una intervención realizada a un grupo de estudiantes del grado segundo de básica primaria de una institución educativa rural del municipio El Zulia (Norte de Santander, Colombia) para el desarrollo de las habilidades comunicativas atendiendo a los estilos de aprendizaje visual, auditivo y kinestésico (VAK).

\section{Metodología}

Se trata de una investigación inscrita en el paradigma histórico hermenéutico-interpretativo y en el enfoque cualitativo dado los métodos e instrumentos utilizados (Hernández, Fernández y Baptista, 2006). La población estuvo conformada por los estudiantes del Centro Educativo Rural Florentino Blanco del municipio de El Zulia y la muestra de la investigación estuvo integrada por 13 estudiantes (7 niños y 6 niñas) del grado segundo de básica primaria, en edades comprendidas entre los 7 y los 10 años. Se escogió este grupo escolar por las falencias que presentan sobre las habilidades comunicativas. El diseño del estudio se enmarca en la denominada investigación-acción (IA). Este tipo de investigación se caracteriza por construir conocimiento a partir de la práctica y su objetivo es resolver los problemas cotidianos o inmediatos, y mejorar las prácticas en concreto (Hernández, et al., 2006).

Tabla 1.

Categorías y dimensiones de análisis de la investigación

\begin{tabular}{lll}
\hline Categoría & Subcategorías & Dimensiones \\
\hline Habilidades & Escribir: Actividad intelectual que se perfecciona y se concreta en un código & Creatividad \\
comunicativas & gráfico para el registro, la comunicación, el control o influir en la conducta de & Exposición de ideas \\
& otros. & Uso adecuado del código \\
& & escrito \\
\cline { 2 - 4 } & Leer: Proceso cognitivo y sociocultural que implica la decodificación del texto & Comprensión \\
& y su ubicación en un contexto específico para su comprensión e & Interpretación \\
& interpretación. & Capacidad crítica
\end{tabular}




\begin{tabular}{ll} 
Escuchar: Capacidad para comprender y reconocer el significado del acto & Atención \\
comunicativo en un determinado hablante-interlocutor. & Tolerancia \\
& Respeto \\
\hline Hablar: Expresión de necesidades, pensamientos, emociones, deseos y & Claridad \\
sentimientos conforme a un código lingüístico en común & Coherencia \\
& Seguridad \\
& Responsabilidad
\end{tabular}

Fuente: Autor

Se utilizaron como instrumentos de recolección de información pruebas para la evaluación de las habilidades comunicativas antes y después de la intervención. Las pruebas iniciales aplicadas a los participantes fueron objeto de una prueba piloto con un grupo de grado segundo de otra sede de la misma institución educativa conformado por 10 estudiantes. Producto de ello se hicieron los cambios respectivos en las pruebas, también se utilizó el diario pedagógico y una prueba para el reconocimiento del sistema o modo de aprendizaje VAK predominante. Las características de estas pruebas se señalan a continuación:

Prueba para la evaluación y diagnóstico de la habilidad comunicativa de escritura (pretest y postest): Esta prueba buscó analizar los aspectos relacionados con la habilidad comunicativa de escritura: creatividad, exposición de ideas y uso adecuado del código escrito. La prueba se estructuró con dos actividades. La primera actividad estuvo diseñada para que el estudiante realizara una producción textual a partir de un binomio de palabras. La segunda actividad buscó que el estudiante describiera hechos de su cotidianidad. En cada actividad se dejó un espacio para la valoración de cada dimensión. Esta prueba se aplicó de forma individual en un tiempo máximo de una hora. La prueba postest mantuvo la misma estructura modificándose los contenidos.

Prueba para la evaluación y diagnóstico de la habilidad comunicativa de lectura: Esta prueba contenía una única actividad en donde el estudiante debía hacer la lectura del texto y a partir del mismo contestar ocho preguntas que iban de menor a mayor complejidad. Cada pregunta buscó analizar los niveles de comprensión, interpretación y capacidad crítica. Se dejó un espacio para la valoración del docente en cada uno de los aspectos analizados. Esta prueba se aplicó de forma individual en un tiempo máximo de una hora. La prueba postest mantuvo la estructura de la prueba inicial modificándose tan sólo los contenidos.

Prueba para la evaluación y diagnóstico de la habilidad comunicativa de escucha y habla: esta prueba estuvo estructurada en tres momentos. En el primer momento el docente organizó grupos de tres estudiantes. A cada grupo el docente le realizó la lectura propuesta y frente a ello valoró los aspectos de la habilidad comunicativa escucha. En el segundo momento, se realizó una pregunta a cada estudiante con el objetivo de valorar la habilidad de habla. Por último, se solicitó a los estudiantes su opinión respecto de las respuestas dadas por los otros dos participantes, valorándose 
de forma integral los aspectos relacionados con estas habilidades.

Diario pedagógico: El diario pedagógico fue aplicado en la implementación y evaluación de un plan diseñado para la mejora de las habilidades comunicativas a partir de los estilos de aprendizaje predominantes. La matriz permitió recopilar los hechos, situaciones, actitudes y comportamientos de los estudiantes en cada una de las actividades diseñadas para mejorar las habilidades comunicativas a través de estrategias en los modos de aprendizaje VAK.

Prueba para el reconocimiento del sistema o modo de aprendizaje VAK predominante: Esta prueba estuvo integrada por seis preguntas y buscó reconocer el modo de aprendizaje más utilizado por cada uno de los estudiantes. Las preguntas de la prueba permiten identificar el modo de aprendizajerepresentación preferido por los estudiantes a partir de situaciones hipotéticas relacionadas con momentos de explicación en el aula, seguimiento de instrucciones, uso de la memoria, entre otros. La prueba aplicada se ha utilizado en otros estudios como el de Silvestre (2009), Cruz y Rodríguez (2012), entre otros. A las primeras cinco preguntas se le dio un valor de $20 \%$ para un total de $100 \%$. La sexta pregunta sirvió para confirmar los hallazgos de las primeras cinco preguntas. Ejemplo del modo de valoración por cada estudiante:

\section{Estudiante 1:}

Pregunta 1. Rta. a

Auditivo $20 \%$

Pregunta 2. Rta. a Auditivo 20\%

Pregunta 3. Rta. c Auditivo $20 \%$
Pregunta 4. Rta. b Auditivo 20\%

Pregunta 5. Rta. c Visual $20 \%$

Pregunta 6. Rta. fAuditivo (Confirma)

Total: $\quad 80 \%$ auditivo y $20 \%$ visual

Una vez recolectada la información a través de la aplicación de los instrumentos y/o técnicas, se diseñó un plan de trabajo a fin de abordar los problemas y dificultades identificadas en el grupo escolar y desde una óptica pedagógica. Dicho plan responde a actividades conforme a los modos de aprendizaje VAK. Para el diseño del plan se tuvo en cuenta los siguientes elementos: objetivos, estrategias, acciones, recursos y programación de tiempos. Con posterioridad, se puso en marcha el plan diseñado. Si bien en el primer momento se recolectó información para definir y diagnosticar el problema, en esta etapa se siguió recolectando datos con el propósito de: (a) revisar los efectos de la implementación del plan, (b) recolectar nuevos datos que permiten reinterpretar el fenómeno, (c) redefinir el problema, y (d) ajustar el plan implementado durante el momento de la intervención para enriquecerlo.

\section{Resultados y discusión}

Estilo de aprendizaje VAK predominante. La Figura 1 muestra por cada estudiante los modos de aprendizaje VAK predominantes y no predominantes. Ninguno de los estudiantes objeto de intervención evidencia un modo de representación predominante de forma absoluta. Por lo general, cada estudiante manifiesta poseer diversos sistemas de aprendizaje, siendo los de 
mayor predominio el auditivo y el visual, y en menor grado el kinestésico. No hay un modo de aprendizaje que no sea utilizado por los estudiantes. Todos los participantes muestran en un mayor o menor grado un predominio de los sistemas de aprendizaje auditivo y visual, y sólo algunos estudiantes no evidencian un desarrollo claro del modo de aprendizaje kinestésico.

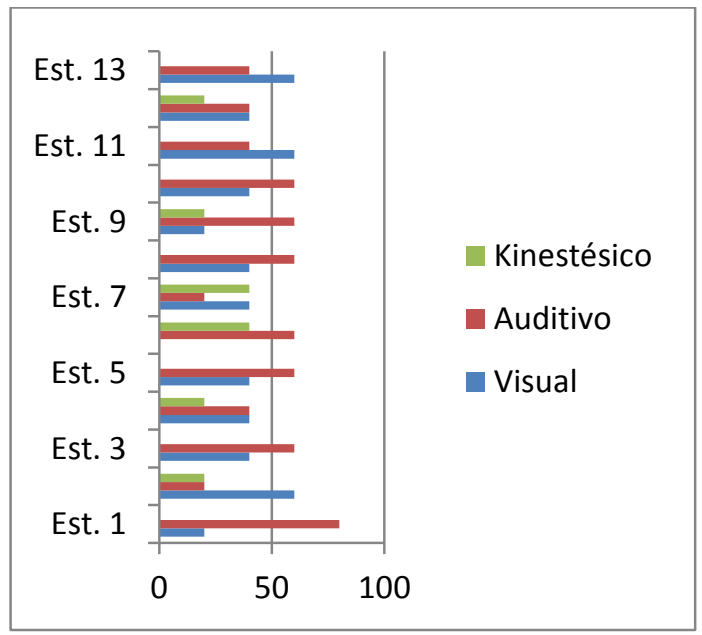

Figura 1. Modo de aprendizaje VAK predominante en los participantes. Fuente: Autor

Diagnóstico de las habilidades comunicativas.

Habilidad escucha. Por lo general, los estudiantes que manifiestan fortaleza en alguna de las variables también lo hacen en los demás criterios de análisis. Lo mismo sucede con los participantes que experimentan deficiencias: si son deficientes en alguna variable también lo son en las demás. Solo los estudiantes 2 y 12 evidencian debilidad en alguno de los criterios y fortalezas en los otros dos, y viceversa. Al comparar estos resultados con los hallazgos de la Gráfica 1 sobre modos de representación VAK predominantes se puede inferir que no hay correlación entre estilos de aprendizaje y fortalezas o deficiencias en la habilidad comunicativa de escucha.

Por otro lado, los estudiantes que muestran fortaleza en la variable de atención se caracterizan por mostrar concentración, comprensión y reconocimiento del código, así como interés y buena disposición. En el caso de los participantes que demuestran una deficiente atención, estos se caracterizan por tener poca concentración y escaso interés. Los estudiantes con fortalezas en la variable de tolerancia muestran una actitud favorable, escuchan de manera respetuosa y están dispuestos a reconocer la opinión de los demás. Los participantes con fortaleza en la variable de respeto evidencian una conducta favorable en donde son tomadas en consideración las reglas de participación como: no interrumpir, solicitar la participación y tener en cuenta los turnos para aportar o preguntar.

Valga aclarar que la prueba aplicada también evaluó la habilidad de escucha al momento de participación de otros compañeros. Los resultados de esta parte infieren que dependiendo de la persona que habla, la habilidad de escucha de los participantes se puede modificar en las variables de atención, tolerancia y respeto. En efecto, cuando el docente toma la palabra y expone ideas, los estudiantes tienden a ser receptivos, pero al momento de escuchar a sus pares, la disponibilidad no es la misma. Por tanto, la figura de autoridad 
influye en la capacidad de escucha de los estudiantes.

Habilidad habla. La claridad se manifiesta en el uso adecuado de las palabras, así como en la cantidad necesaria para exponer una idea. La coherencia evaluó la habilidad del estudiante para ser congruente con las preguntas, así como la identidad entre la opinión de los otros y sus opiniones. Del mismo modo, la coherencia se observó en la habilidad del sujeto para dar respuestas que infirieran el reconocimiento de la intención del autor del texto. La seguridad se observó a través del tono utilizado al hablar y la actitud. $Y$ a través del criterio de responsabilidad se examinó la capacidad de los sujetos para: i) ser prudente al momento de hablar, ii) no ofender a los otros y iii) estar atento a la discusión a fin de aportar a la discusión.

En cuanto a los resultados obtenidos se puede observar que las fortalezas o deficiencias se presentan de manera global, excepto en los casos de los estudiantes 3 y 4 . La mayoría de los estudiantes presentan fortalezas en cada una de las variables analizadas, aunque un número considerable de estudiantes se caracterizan por no ser claros al hablar, bien por dificultades del lenguaje (v.g. uso de combinaciones) o bien por la misma inseguridad que los bloquea. También se puede reconocer que la mayoría de los estudiantes son coherentes al momento de ofrecer respuestas demostrando reconocer la intencionalidad de la pregunta y la intención del autor. También un número significativo de estudiantes se caracterizan por ser prudentes al hablar, estar atentos a la discusión, respetar normas y reglas de participación.

Al comparar estos resultados con los modos de representación predominantes (Gráfico 1) se logra establecer una relación entre debilidad global en la habilidad de habla y el sistema de representación predominante auditivo. En otras palabras, estos estudiantes tienen un mejor aprendizaje escuchando al docente, pero ello no es garantía de un desarrollo adecuado de la habilidad comunicativa de habla. Por otro lado, también se evidencia una correlación entre estos resultados y los hallazgos de la Tabla 3. Los estudiantes que manifiestan una deficiencia global en la habilidad de habla, también presentan dificultades en las variables asociadas con la habilidad de escucha.

Habilidad lectura. La comprensión se refiere a la habilidad del sujeto para reconocer datos e información en el texto permitiéndole construir una idea global del mismo. El estudiante es capaz de narrar o dar razón de aquello que ha sido expuesto en el texto. Por otro lado, la interpretación se ve reflejada en la habilidad para establecer relaciones entre dos o más tipos de información generando inferencias o conclusiones. Por último, la criticidad es la capacidad para identificar la posición e intención del autor del texto y contextualizar el mensaje. Como se ve reflejado en los resultados del pre-test, la mayoría de los estudiantes comprenden el texto. Esto se manifiesta en respuestas básicas relacionadas con los personajes del texto o hechos específicos que se 
palabras y oraciones, y a su intencionalidad. En este punto se reconoce el estado del proceso escritor en el que se encuentra el niño o la niña: garabateo (icónico-no icónico), silábico o arribo a la escritura alfabética.

Los hallazgos muestran que las principales falencias se encuentran en la exposición de ideas y el uso del código. La mayoría de los niños deberían encontrarse en un proceso de arribo a la escritura alfabética construyendo palabras y oraciones precisas. Sin embargo, más de la mitad del grupo se encuentran en etapas anteriores. Algunas manifestaciones son: confusión de signos y fonemas, no establecer correspondencias entre fonemas y grafemas, no utilizar signos de puntuación, demostrar variadas deficiencias ortográficas, entre otros.

Algunos participantes no logran exponer ideas de forma clara y precisa percibiéndose incoherencia o incapacidad para terminar de desarrollar hechos o situaciones propuestas. Estas deficiencias tienen su origen en varios factores como escasez de instrumentos verbales, el sentimiento de frustración y baja competencia al observar que no puede hacer uso del código escrito y al poco desarrollo de espacios para la producción de textos de forma libre y autónoma.

Intervención por sesión. Descripción de las actividades

Tres estudiantes que presentaban dificultades de forma global en cada una de las variables de la habilidad comunicativa escucha, ahora evidencian 
fortalezas. Asimismo, dos estudiantes evidencian cambios parciales en las variables de respeto y atención. Estos resultados son favorables pues la aplicación de estrategias didácticas basadas en modos de aprendizaje VAK direcciona las acciones pedagógicas del docente hacia propósitos concretos. La eficacia de las actividades pedagógicas no radica en los recursos didácticos diseñados sino en las relaciones de comunicación que se establecen entre docente-estudiantes y estudiantes-pares. Estas relaciones se establecen con base en reglas específicas en donde se promociona la atención, el respeto, la tolerancia, la ayuda mutua y el aprendizaje colaborativo.

En cuanto a la habilidad del habla se observan cambios significativos en cuatro estudiantes y modificaciones parciales en tres. La mejora de los procesos de comunicación permite un mayor desarrollo de estas habilidades. También al generarse un espacio de mayor respeto, tolerancia y atención, los estudiantes enfrentan con mayor agrado los retos impuestos al momento de hablar disminuyendo la timidez y generando mayor confianza entre los participantes. Los estudiantes que mejoraron esta habilidad ahora utilizan tonos de voz adecuados, muestran coherencia al exponer ideas, transmiten seguridad y argumentan sus respuestas. Siete estudiantes presentaron cambios y ahora demuestran fortaleza en variables que en una etapa inicial evidenciaban serias limitaciones o debilidades en lectura. Los principales cambios se reflejan en las variables de interpretación y criticidad. Una de las razones por las cuales se presentaron estos cambios es la reducción del nivel de presión entre los estudiantes y el aumento del sentimiento de competencia en los participantes. La lectura conforma un proceso complejo que implica exploración, formulación de hipótesis, establecimiento de relaciones para otorgar valores sonoros, entre otros. Diferentes actos interiorizados a nivel de pensamiento se encuentran relacionados con este proceso: identificación, diferenciación, comparación y análisis. Cada una de estas operaciones se fortalecieron con el desarrollo de las acciones pedagógicas, y el diseño de las actividades basadas en los modos de aprendizaje VAK facilitaron estos resultados. El uso de estrategias visuales y kinestésicas promueven el desarrollo de la capacidad de interpretación y la capacidad crítica. En efecto, los estudiantes tenían oportunidad de visualizar videos, participar de puestas en escenas, discutir los textos, entre otras acciones propias del aprendizaje significativo y el aprendizaje colaborativo. La escritura es la habilidad comunicativa que evidencia mayores cambios al compararse estos resultados con los hallazgos de la prueba inicial. Díez estudiantes evidencian en diferentes variables una mejoría notoria. Del mismo modo, otros estudiantes que ya presentaban fortaleza en algunas de las variables demostraron un mayor desarrollo de la habilidad. La producción de textos mejoró de forma global entre los participantes. A diferencia de los resultados iniciales, los estudiantes muestran una mayor creatividad al momento de escribir y por ello se observan textos más amplios con ideas más elaboradas y desarrolladas (Figura 2). 
Tabla 3. Intervención por sesión. Descripción de las actividades

\begin{tabular}{|c|c|}
\hline Sesión & Descripción \\
\hline 1 & $\begin{array}{l}\text { VAK: Visual-auditivo } \\
\text { HC.: Escuchar, escribir } \\
\text { Contenido: El poema } \\
\text { Actividad: Se motivó a los estudiantes por medio del modo de aprendizaje visual- auditivo, a través de la } \\
\text { observación del video del poema "La pobre viejecita". }\end{array}$ \\
\hline 2 & $\begin{array}{l}\text { VAK: Visual-kinestésico } \\
\text { HC: Escuchar, hablar, leer. } \\
\text { Contenido: El poema } \\
\text { Actividad: Se trabajó bajo el modo de aprendizaje visual y kinestésico, dando continuidad a la anterior sobre el } \\
\text { poema "La pobre viejecita”. Se buscó desarrollar las habilidades comunicativas, escuchar, hablar y leer. Esto } \\
\text { permitió hacer un paralelo entre las dos actividades. }\end{array}$ \\
\hline 3 & $\begin{array}{l}\text { VAK: Visual auditivo-kinestésico } \\
\text { HC: leer, escuchar, hablar } \\
\text { Contenido: Teatro } \\
\text { Actividad: Se trabajó bajo el modo de aprendizaje visual auditivo y kinestésico. Se buscó desarrollar las habilidades } \\
\text { comunicativas, leer, escuchar y habar. Esta actividad permitió afianzar procesos significativos para los estudiantes } \\
\text { a través del texto "El renacuajo paseador". }\end{array}$ \\
\hline 4 & $\begin{array}{l}\text { VAK: Visual- kinestésico } \\
\text { HC: Leer, escribir Contenido: el poema. } \\
\text { Actividad: Se da continuidad a la anterior actividad, buscando desarrollar las habilidades comunicativas leer y } \\
\text { escribir. Se trabajó bajo el modo de aprendizaje visual y kinestésico. }\end{array}$ \\
\hline 5 & $\begin{array}{l}\text { VAK: Visual-kinestésico. } \\
\text { HC: Leer, escribir. Contenido: El antónimo } \\
\text { Actividad: Se hizo uso de material concreto manipulativo, en el cual los estudiantes debían recortar láminas con } \\
\text { imágenes y su respectiva palabra o significado. Se buscó desarrollar las habilidades leer y escribir a través del } \\
\text { modo de aprendizaje visual y kinestésico. }\end{array}$ \\
\hline 6 & $\begin{array}{l}\text { VAK: Visual-auditivo } \\
\text { HC: Leer, escuchar, hablar Contenido: La descripción } \\
\text { Actividad: Esta actividad se hizo bajo el modo de aprendizaje visual auditivo, logrando captar la atención de los } \\
\text { estudiantes para una mayor motivación. }\end{array}$ \\
\hline 7 & $\begin{array}{l}\text { VAK: Visual-auditivo-kinestésico } \\
\text { HC: Leer, escribir } \\
\text { Contenido: La descripción } \\
\text { Actividad: Esta actividad se desarrolló de acuerdo con lo trabajado en la actividad 6, empleando el modo de } \\
\text { aprendizaje visual-auditivo-kinestésico, en busca a desarrollar las habilidades comunicativas leer y escribir. }\end{array}$ \\
\hline 8 & $\begin{array}{l}\text { VAK: Visual, auditivo } \\
\text { HC: Escuchar, escribir } \\
\text { Contenido: La fábula } \\
\text { Actividad: Se empleó el modo de aprendizaje visual-auditivo, a través del video “La cigarra y la hormiga” (fábula). } \\
\text { Se buscó desarrollar las habilidades comunicativas escuchar-escribir. }\end{array}$ \\
\hline 9 & $\begin{array}{l}\text { VAK: Auditivo-kinestésico } \\
\text { HC: Leer, escuchar, hablar Contenido: El poema dramatizado } \\
\text { Actividad: Se desarrolló bajo el modo de aprendizaje auditivo- kinestésico, a fin de desarrollar las habilidades } \\
\text { comunicativas leer, escuchar y hablar. }\end{array}$ \\
\hline 10 & $\begin{array}{l}\text { VAK: Visual, auditivo, kinestésico } \\
\text { HC: Leer, escribir. Contenido: El poema } \\
\text { Actividad: Para esta actividad los estudiantes ya tenían conocimientos previos al tema. Se buscó desarrollar la } \\
\text { habilidad de lectura y escritura. }\end{array}$ \\
\hline 11 & $\begin{array}{l}\text { VAK: Visual, auditivo-kinestésico } \\
\text { HC: Leer, escribir Contenido: La leyenda } \\
\text { Actividad: La actividad fue desarrollada desde el modo de aprendizaje visual, auditivo y kinestésico. Se buscó } \\
\text { desarrollar las habilidades de lectura y escritura. }\end{array}$ \\
\hline 12 & $\begin{array}{l}\text { VAK: Visual-kinestésico } \\
\text { HC: Lectura, escritura } \\
\text { Actividad: En esta actividad se busca desarrollar las habilidades leer y escribir a partir de los modos de aprendizaje } \\
\text { visual-kinestésico. }\end{array}$ \\
\hline
\end{tabular}

Fuente. Autor 
El docente se muestra como un actor clave que media entre el estímulo, el organismo y su respuesta. La contribución del docente es fundamental para el desarrollo de habilidades comunicativas. Los resultados muestran cambios favorables pues se presentó un desarrollo global de las competencias relacionadas con las diferentes habilidades comunicativas escuchar, hablar, leer y escribir.

\section{Referencias}

Alonso, C. M., Gallego, D. J., y Honey, P. (2007). Los estilos de aprendizaje: Procedimientos de diagnóstico y mejora. Bilbao, España: Editorial Mensajero.

Aragón, M. y Jiménez, Y. (2009). Diagnóstico de los estilos de aprendizaje en los estudiantes: Estrategia docente para elevar la calidad educativa. CPU-e Revista de Investigación Educativa, (9), 121.

Basanta, A. (2005). La pasión de leer. Revista de Educación, 189-201. Bravo, L., Villalón, M., y Orellana, E. (2002). La conciencia fonológica y la lectura inicial en niños que ingresan a primer año básico. Psykhe, 11(1), 175-182. Burón, J. (1996). Enseñar a aprender: Introducción a la metacognición (p. 160). Bilbao, España: Ediciones Mensajero.

Camps, A. (2005). Hablar en clase, aprender lengua. En E. Larreula, J. Ferrer, L. López, M. Rodeiro, L. Nussbaum, M. U. Bikandi, ... M. Vilá.
Hablar en clase: cómo trabajar la lengua oral en el centro escolar (pp. 37-46). Barcelona, España: Graó.

Cassany, D., Luna, M., y Sanz G. (1999). Enseñar lengua (p. 151). Barcelona, España: Grao.

Cruz, S., y Rodríguez, J. (2012). Pistas de quiénes son y cómo aprenden los estudiantes de la Escuela de Ciencias de la Comunicación Colectiva de la Universidad de Costa Rica. Reflexiones, (34), 3343.

Díez, C. (1999). La interacción en el inicio de la lectoescritura (p. 140). Madrid, España: Ministerio de Educación y Cultura. Ferreiro, E., y Teberosky, A. (1998). Los sistemas de escritura en el desarrollo del niño (p. 367). Ciudad de México, México: Siglo XXI.

Hernández, R., Fernández, C., y Baptista, MdelP. (2006). Metodología de la investigación (p. 850). Ciudad de México, México, McGraw-Hill.

Kalman, J. (2008). Discusiones conceptuales en el campo de la cultura escrita. Revista Iberoamericana de Educación, (46), 107-134.

Ministerio de Educación Nacional. (1998). Lineamientos Curriculares de Lengua Castellana, Bogotá, Colombia: Ministerio de Educación Nacional.

Ocaña, J. A. (2010). Mapas mentales y estilos de aprendizaje. (Aprender a cualquier edad) (p. 295). Alicante, España: ECU. 
Romo, M. E., López, D., y López, I. (2006). ¿Eres visual, auditivo o kinestésico? Estilos de aprendizaje desde el modelo de la programación neurolingüística (PNL). Revista Iberoamericana de Educación, 38(2), 1-9.

Ruíz, G. A. (2010). La cognición y la metacognición: dos formas complementarias de entender la lectura y la escritura. Revista Visión Contable, (8), 97-122. Solé, I. (1994). Aprender a usar la lengua: Implicaciones para la enseñanza. Aula de Innovación Educativa, (26), 5-10.

Tolchinsky, L. (1993). Aprendizaje del lenguaje escrito: procesos evolutivos e implicaciones didácticas. Barcelona: Anthropos.

Valderrama, C. E. (2000). Comunicacióneducación: coordenadas, abordajes y travesías $(p$. 426). Bogotá, Colombia: Siglo del Hombre Editores. Vygotski, L. (1978). El desarrollo de los procesos psicológicos superiores (p. 224). Barcelona, España: Editorial Crítica. 